\title{
WILEY-VCH
}

DOI: 10.1002/ ((please add manuscript number))

Article type: Full Paper

\section{Size-controlled Si nanocrystals fabricated by electron beam evaporation}

O. Blázquez, L. López-Conesa, J. López-Vidrier, J. L. Frieiro, S. Estradé, F. Peiró, J. Ibáñez, S. Hernández, ${ }^{*}$ B. Garrido

O. Blázquez, Dr. L. López-Conesa, J. L. Frieiro, Dr. S. Estradé, Prof. F. Peiró, Dr. S. Hernández, Prof. B. Garrido MIND, Departament d'Enginyeria Electrònica i Biomèdica, Universitat de Barcelona, Martí i Franquès 1, E-08028 Barcelona, Spain.

Institute of Nanoscience and Nanotechnology (IN2UB), Universitat de Barcelona, Av. Joan XXIII S/N, E-08028 Barcelona, Spain.

E-mail: shernandez@ub.edu, oblazquez@el.ub.edu

Dr. J. López-Vidrier

Laboratory for Nanotechnology, Dept. of Microsystems Engineering (IMTEK), University of Freiburg, Albert-Ludwigs-University Freiburg, Georges-Köhler-Allee 103, D-79110 Freiburg, Germany.

Dr. J. Ibáñez

Institute of Earth Sciences Jaume Almera, ICTJA-CSIC, Lluís Solé i Sabarís s/n, 08028

Barcelona, Catalonia, Spain

Keywords: Multilayered silicon nanocrystals, electron beam evaporation, transmission electron microscopy, Raman scattering, photoluminescence

Multilayers consisting of silicon nanocrystals ( $\mathrm{Si} \mathrm{NCs}$ ) and $\mathrm{SiO}_{2}$ have been successfully fabricated by electron beam evaporation, using pure $\mathrm{Si}$ and $\mathrm{SiO}_{2}$ targets in an oxygen-rich atmosphere for alternately depositing silicon-rich oxide (SRO) layers and $\mathrm{SiO}_{2}$ barriers, respectively. A post-deposition annealing process was carried out at different temperatures in order to achieve the Si precipitation in the form of nanocrystals. The stoichiometry of the layers was determined by X-ray spectroscopy, which confirmed the controlled silicon oxidation in order to attain SRO layers. Transmission electron microscopy and Ramanscattering measurements confirmed the presence of crystalline Si-nanoprecipitates. Photoluminescence spectra from the Si NC samples could be deconvolved into two contributions, whose dynamics suggests that two different luminescent centres are responsible for the optical emission of the samples. 


\section{WILEY-VCH}

\section{Introduction}

Since the middle of the last century, silicon has been one of the most important semiconductor materials studied by researchers thanks to its abundance, non-toxicity and good electrical properties, thus being widely employed in the electronics industry. However, its indirect band gap is the main drawback for optical applications, which drastically decreases the probability of electron-hole radiative recombination. As it is well known, nanostructuring Si results in the quantization and energy increase of its allowed electronic states, properties that strongly depend on the nanostructure size. ${ }^{[1-3]}$ The consequent tunability of the band gap of $\mathrm{Si}$ nanocrystals (Si NCs) has been widely investigated in order to employ their optical properties in light-emitting, ${ }^{[4-7]}$ photovoltaic ${ }^{[8-10]}$ devices or memory applications. ${ }^{[1,12]}$ Matrixembedded $\mathrm{Si} \mathrm{NCs}$ are one of the most employed configurations, being $\mathrm{SiO}_{2}$ the most usual dielectric matrix. By controlling the stoichiometry of silicon oxide (i.e., the Si excess), a hightemperature annealing process can be carried out which promotes the precipitation of the $\mathrm{Si}$ excess in the form of Si NCs.

On one hand, the control of the $\mathrm{Si} \mathrm{NC}$ size has been achieved by the $\mathrm{Si} \mathrm{NCs} / \mathrm{SiO}_{2}$ multilayers (MLs) approach, where the stoichiometric $\mathrm{SiO}_{2}$ layers play the role of barrier against $\mathrm{Si}$ diffusion, thus limiting the growth of the Si NCs along the vertical direction. ${ }^{[1]}$ In addition, several works on the effect of the annealing temperature $\left(T_{\mathrm{a}}\right)$ and the thickness of the $\mathrm{SiO}_{2}$ barriers have been carried out to determine the optimal structure for optoelectronics applications. ${ }^{[13,14]}$ On the other hand, the fabrication of these nanostructures has basically been achieved by means of either chemical-vapor deposition, ${ }^{[3,15]}$ sputtering, ${ }^{[16]}$ or Si implantation in $\mathrm{SiO}_{2} \cdot{ }^{[17]}$ The electron-beam evaporation (EBE) technique has also been employed to generate multilayered $\mathrm{Si} \mathrm{NCs} / \mathrm{SiO}_{2}$ structures. However, whereas the $\mathrm{SiO}_{2}$ layers can be easily EBE-deposited, the silicon-rich oxide (SRO) layers are typically achieved via either thermal 


\section{WILEY-VCH}

evaporation $^{[18]}$ or $\mathrm{EBE}^{[19]}$ of $\mathrm{SiO}$ targets. So far, the possibility to fabricate $\mathrm{Si} \mathrm{NCs}$ evaporating both $\mathrm{SiO}_{2}$ and $\mathrm{SRO}$ layers employing the same EBE technique has not been explored yet. In the case of SRO, using pure Si target provides an alternative techniquecompatible strategy; indeed, this has proved useful to synthesize colloidal Si NCs by placing the pure Si target in deionized water, followed by a thermal treatment via ultra-short pulsed laser ablation. ${ }^{[20]}$

In this work, we present the fabrication, entirely carried out by means of the EBE technique, as well as the structural and optical characterization of $\mathrm{Si} \mathrm{NCs}$ embedded in a $\mathrm{SiO}_{2}$ matrix. For this, a ML approach was employed by alternating the deposition of SRO layers and $\mathrm{SiO}_{2}$ barriers, respectively using pure $\mathrm{Si}$ and $\mathrm{SiO}_{2}$ targets in an oxygen-rich atmosphere.

\section{Results and discussion}

First, we study the stoichiometry of $\mathrm{SiO}_{2}$ and $\mathrm{SRO}$ layers deposited by e-beam and annealed at $1200{ }^{\circ} \mathrm{C}$ by X-ray photoelectron spectroscopy (XPS). For this analysis, due to the limited depth resolution of the experimental set-up (during the sputtering process), bulk $\mathrm{SiO}_{2}$ and SRO samples were employed for determining their composition, and thus the equivalent on of the ML samples. Figure 1 shows the Si2p and O1s XPS signals, whose areas under the curve were determined and corrected for the sensitivity factor of each atomic specimen. From this analysis, atomic concentrations of $\mathrm{Si}(34 \%)$ and $\mathrm{O}(66 \%)$ were determined for the $\mathrm{SiO}_{2}$ sample, which confirms its stoichiometry. In contrast, the SRO layer presents a clear substoichiometry with atomic values of $\mathrm{Si}(42 \%)$ and $\mathrm{O}(58 \%)$, corresponding to $\mathrm{SiO}_{1.4}$, which confirms that Si is partially oxidized when it is evaporated from a pure Si target with an $\mathrm{O}_{2}$ line. Exploring in more detail the $\mathrm{Si} 2 \mathrm{p}$ spectra of both $\mathrm{SiO}_{2}$ and $\mathrm{SRO}$ layers, deconvolution via different pseudo-Voigt fits was performed in order to determine the contribution of the different oxidation states of $\mathrm{Si}$. In the case of the $\mathrm{SiO}_{2}$ sample [see Figure 1(a)], the main contribution corresponds to the $\mathrm{Si}^{4+}$ state $(103.7 \mathrm{eV})$, with a decreasing contribution of the 


\section{WILEY-VCH}

other oxidation states as the oxidation number decreases, namely $\mathrm{Si}^{3+}(102.6 \mathrm{eV}), \mathrm{Si}^{2+}(101.4$ $\mathrm{eV}$ ) and $\mathrm{Si}^{1+}(100.1 \mathrm{eV})$, progressively. No contribution of $\mathrm{Si}^{0}$ is observed, which is in agreement with the stoichiometry of this layer. On the other hand, Figure 1(b) shows the Si2p signal of the SRO layer, exhibiting a broader spectrum than the $\mathrm{SiO}_{2}$ layer. In this case, the distribution of the different oxidation states of silicon is clearly changed, decreasing the $\mathrm{Si}^{4+}$ $(103.7 \mathrm{eV})$ contribution and increasing the $\mathrm{Si}^{3+}(102.5 \mathrm{eV}), \mathrm{Si}^{2+}(101.3 \mathrm{eV})$ and $\mathrm{Si}^{1+}$ $(100.0 \mathrm{eV})$ ones. In contrast to the $\mathrm{SiO}_{2}$ layer, the $\mathrm{SRO}$ layer shows the metallic $\mathrm{Si}^{0}(98.6 \mathrm{eV})$ contribution, indicating Si-Si bonds due to the lack of oxygen. Regarding the O1s analysis of the $\mathrm{SiO}_{2}$ presented in Figure 1(c), a single pseudo-Voigt function was fitted at $532.0 \mathrm{eV}$, indicating that oxygen is only bound to $\mathrm{Si}$ atoms in a single configuration (-O-). However, the SRO layer exhibits an O1s broader spectrum [see Figure 1(d)], which suggests that part of the oxygen presents different bonds. The main contribution is centered at $532.1 \mathrm{eV}$ and it is again attributed to oxygen bound to $\mathrm{Si}$ atoms in single configuration (-O-), like within the $\mathrm{SiO}_{2}$ layer. The other contribution displays a higher energy $(533.6 \mathrm{eV})$ and it can be directly correlated with the substoichiometry of the layer. In this case, peroxy configuration (-O-O-) is sensitive to be formed in the regions where Si excess is present. ${ }^{[21]}$ In Table I, the binding energy, integrated area and resulting atomic concentration of each contribution are summarized.

Transmission electron microscopy (TEM) images of the test sample annealed at $1200{ }^{\circ} \mathrm{C}$ were analyzed in order to match the nominal thicknesses with the measured Si NC sizes. In the Figure 2(a), an energy-filtered TEM (EFTEM) image of this sample is exhibited, filtering the energy around the Si plasmon peak $(\sim 17 \mathrm{eV})$. The image clearly confirms the ML structure even at this high annealing temperature, where the brightest zones correspond to a higher $\mathrm{Si}$ concentration. In the stack near the Si substrate (the first layers at the bottom of the image), the $\mathrm{SiO}_{2}$ barriers are kept constant at a measured thickness of $2.7 \mathrm{~nm}$, while the measured 


\section{WILEY-VCH}

thickness of the SRO layers varies from $3.5 \mathrm{~nm}$ to $4.7 \mathrm{~nm}$. In the second stack (layers at the top of the image), the $\mathrm{SiO}_{2}$ barriers exhibit thickness values that vary from $2.3 \mathrm{~nm}$ to $3.5 \mathrm{~nm}$, with constant SRO layers of a measured thickness of $3.5 \mathrm{~nm}$. Thanks to this test sample, nominal and real thicknesses were correlated. Indeed, the increase in nominal thickness clearly corresponds to an increase in the real (measured) thickness. This relation between nominal and measured $\mathrm{SiO}_{2}$ and $\mathrm{SRO}$ thicknesses is plotted in Figure 2(b), displaying a linear trend. This study has allowed calibrating our deposition set-up and determining the real thickness of the SRO layers $\left(L_{\mathrm{SRO}}\right)$. High-resolution TEM (HRTEM) images were also acquired in the center of this first stack to determine whether Si NC formation was achieved during the annealing process. In Figure 2(c), different Si lattice planes (highlighted by red lines) are observed and attributed to the $\mathrm{Si} \mathrm{NC}$ formation, providing an unequivocal proof that the Si nanoaggregates contain a non-negligible crystalline phase. It is important to note that only crystalline planes parallel to the electron-beam can be observed, which substantially reduces the number of visible Si NCs.

Raman measurements were performed at room temperature on samples deposited on fused silica substrates. In Figure 3(a), the Raman spectra corresponding to the sample with the largest SRO thickness $\left(L_{\mathrm{SRO}}=4.7 \mathrm{~nm}\right)$, annealed at different $T_{\mathrm{a}}$, are plotted. Two different features can be clearly distinguished from the spectra: a broad band from $300 \mathrm{~cm}^{-1}$ to $500 \mathrm{~cm}^{-}$ 1, with its maximum around $470 \mathrm{~cm}^{-1}$, related to disorder-activated acoustical and optical modes in amorphous $\mathrm{Si}$ and amorphous $\mathrm{SiO}_{2}$, and a peak centered around $\sim 517 \mathrm{~cm}^{-1}$, attributed to the crystalline Si transversal-longitudinal optical (TO-LO) phonon mode. The first band is observed in all the spectra, whereas the crystalline feature appears only after an annealing beyond $1000^{\circ} \mathrm{C}$. This last feature indicates the formation of crystalline aggregates under these particular thermal annealing conditions. Despite the fact that the precipitation of the Si excess within the SRO layers and the formation of amorphous Si nanoclusters already 


\section{WILEY-VCH}

takes place at temperatures as low as $700-800{ }^{\circ} \mathrm{C}$, a higher $T_{\mathrm{a}}$ is necessary for the crystallization of these nanoaggregates. ${ }^{[13,22]}$ In this regard, the presence of the crystalline $\mathrm{Si}$ peak in the spectra corresponding to samples annealed at $T_{\mathrm{a}}>1000{ }^{\circ} \mathrm{C}$ confirms the presence of a Si crystalline phase, which totally agrees with the lattice planes observed by HRTEM [see Figure 2(c)].

The Raman spectra from the samples annealed at $1200{ }^{\circ} \mathrm{C}$ and containing different $L$ SRO are displayed in Figure 3(b), showing the above-mentioned contributions. However, in this case, the peak related to crystalline $\mathrm{Si}$ is only observed for $L_{\mathrm{SRO}}$ equal or thicker than $4.3 \mathrm{~nm}$, with no observation of thinner SRO layers. In fact, the observed contribution from the $\mathrm{SiO}_{2}$, either coming from the surroundings or from the substrate, could be masking the crystalline contribution. Another possible explanation might be ascribed to the fact that a critical Si mass is necessary to achieve the formation of crystalline aggregates. ${ }^{[23]}$ Analyzing this crystalline feature using the phonon confinement model described in Ref. 24 in samples with $L_{\mathrm{SRO}}=$ $4.3 \mathrm{~nm}$ and $L_{\mathrm{SRO}}=4.7 \mathrm{~nm}$ [see Figure 3(b)], we have found a crystalline $\mathrm{Si}$ NC size of 3.9 and $4.2 \mathrm{~nm}$, respectively. In addition, by comparing the total Si-cluster size from TEM and the Sicrystalline size from the Raman analysis, we conclude that there is an almost full crystallization of the precipitated $\mathrm{Si}$, in good accordance with previous results in a similar system. ${ }^{[24]}$ These results are in excellent agreement with HRTEM images, where crystalline nanoaggregates are observed with sizes around $4 \mathrm{~nm}$ [see Figure 2(c)].

Photoluminescence measurements were carried out on EBE-grown $\mathrm{Si} \mathrm{NCs} / \mathrm{SiO}_{2}$ samples deposited on Si substrates, exciting them with the 532-nm line of a continuous wave Nd:YAG laser. In Figure 4(a), the PL spectra from the samples with $L_{\mathrm{SRO}}=3.9 \mathrm{~nm}$ and annealed at different temperatures are shown. In the case of the as-deposited sample, no emission is observed, which is in agreement with the fact that the precipitation of the Si excess in the form of nanoaggregates has not taken place yet. In contrast, annealed samples exhibit PL 


\section{WILEY-VCH}

emission with a broad spectrum in the red-infrared part of the spectrum. The intensity of this PL emission increases with the annealing temperature, achieving its maximum at $1200{ }^{\circ} \mathrm{C}$. A similar evolution has been found for the samples with different $L_{S R O}$, indicating that the optimum annealing temperature for obtaining improved optical emission matches with the one for having high crystalline quality, as demonstrated from the Raman study.

The PL spectra of the samples with different $L_{\mathrm{SRO}}$ and annealed at $1200{ }^{\circ} \mathrm{C}$ are shown in Figure 4(b). In this case, the PL intensity decreases as the $L_{\text {SRO }}$ increases, exhibiting also a broader lineshape. Considering that the PL lineshape from Si NCs is constituted by a combination of Gaussian contributions (see for instance Refs. 19 and 25), our spectra could be deconvolved using only two Gaussian functions in the energy domain, one of them centered at higher energies (around $750 \mathrm{~nm}$ ) and another centered at lower energies (around $900 \mathrm{~nm}$ ). The deconvolution of the PL spectra for samples with $L_{\mathrm{SRO}}=3.5 \mathrm{~nm}$ and $L_{\mathrm{SRO}}=4.7 \mathrm{~nm}$ is displayed in Figure 4 (c), exhibiting excellent accordance between the experimental spectra and the Gaussian fits. In Figure 4(d), the band gap energy corresponding to each contribution as a function of $L_{\mathrm{SRO}}$ is plotted for all samples annealed at $T_{\mathrm{a}}=1200^{\circ} \mathrm{C}$. The peak energy of both contributions decreases for larger $L_{\mathrm{SRO}}$ values, which is consistent with the reduction of quantum confinement of carriers in larger Si NCs. However, this trend is more accentuated in the case of the lower-energy contribution (green circles). In addition, the deconvolved peak at lower energies displays an overall intensity which is almost independent on $L_{\mathrm{SRO}}$, in contrast to the intensity of the higher-energy contribution (blue squares), exhibiting a pronounced intensity quenching at larger $L_{\text {SRO }}$ [see Figure $4(\mathrm{e})$ ]. Considering the peak energy values and

the intensity behavior reported in the literature for Si NCs with similar sizes, ${ }^{[1-3,25]}$ our observations suggest that the low-energy contribution is directly related to $\mathrm{Si}$ NCs emission via radiative electron-hole recombination, whereas the high energy contribution could be 


\section{WILEY-VCH}

associated to highly-localized defect levels at the $\mathrm{Si}_{-} \mathrm{SiO}_{2}$ interface, in good agreement with Ref. 25.

In order to obtain more information about the origin of both contributions, PL dynamics was studied by exciting the samples with a $\mathrm{Nd}^{3+}$ :YAG 5-ns-pulsed laser. To perform time-resolved PL spectroscopy, the monochromator was set at two different wavelengths, $850 \mathrm{~nm}$ and 650 nm, which have been selected to separately maximize each PL contribution. The PL time decay of the samples annealed at $1200{ }^{\circ} \mathrm{C}$ for different $L_{\mathrm{SRO}}$, at $850 \mathrm{~nm}$ and $650 \mathrm{~nm}$, is shown in Figure 5(a) and 5(b), respectively. At both wavelengths, the behavior of the PL dynamics suggests two different time evolutions, which could be fitted by the combination of two stretched exponential decay functions, following the typical analysis for Si NCs: ${ }^{[26]}$

$$
I_{\mathrm{PL}}=A_{1} \exp \left[-\left(\frac{t}{\tau_{1}}\right)^{\beta_{1}}\right]+A_{2} \exp \left[-\left(\frac{t}{\tau_{2}}\right)^{\beta_{2}}\right]
$$

The first term in Equation 1 represents the PL decay with a faster dynamics, characterized by its decay time $\tau_{1}$, stretching parameter $\beta_{1}$ and amplitude $A_{1}$; the second term describes the slower PL decay, with its own decay time $\tau_{2}$, amplitude $A_{2}$, and stretching parameter $\beta_{2}$. In all cases, the faster contribution exhibits a stretching parameter $\beta_{1}=1$ (i.e., equivalent to a single exponential) and a decay time of $\tau_{1}=(140 \pm 10) \mathrm{ns}$, which matches with time response limitations of our experimental set-up. Hence, there are luminescent centers with decay time faster than this value (and thus not measurable by our equipment), which are typically attributed to emission related to $\mathrm{SiO}_{2}$ matrix defects (probably due to oxygen vacancies). ${ }^{[27-29]}$ The slow decay time $\tau_{2}$ exhibits a different behavior depending on the observation wavelength. Comparing Figure 5(a) and 5(b), one can observe that the low-energy contribution at $850 \mathrm{~nm}$ presents decay times $\tau_{2}$ lower than the high-energy one at $650 \mathrm{~nm}$, which suggests a different nature of the luminescent centers. In addition, the time evolution of $\mathrm{PL}$ at $850 \mathrm{~nm}$ shows a lower stretching parameter than the curves at $650 \mathrm{~nm}$. In order to study 


\section{WILEY-VCH}

these parameters in detail, the evolution of $\tau_{2}$ and $\beta$ as a function of $L_{\mathrm{SRO}}$ are represented for both contributions [Figures 5(c) and 5(d)]. The Figure 5(c) shows that the contribution at 850 nm shows an increase of $\tau_{2}$ for larger $L_{\mathrm{SRO}}$, obtaining a constant value at $L_{\mathrm{SRO}}$ larger than 4.3 $\mathrm{nm}$, whereas the $\beta$ value slowly increases with $L_{\mathrm{SRO}}$. Analyzing both parameters $\left(\tau_{2}\right.$ and $\left.\beta\right)$ for the high-energy contribution [see Figure 5(d)], one can see that $\tau_{2}$ exhibits values around one order of magnitude lower than the low-energy contribution, as well as decreasing at thicker $L_{\text {SRO. }}$ Again, the stretching parameter $\beta$ exhibits the same evolution as a function of $L_{\mathrm{SRO}}$ than in the low-energy contribution, in which a clear increase of its value is observed, despite still keeping lower values.

The results concerning the excitonic recombination dynamics can be further discussed in terms of their time evolution. In particular, the stretched exponential behavior exhibited by both low- $(850 \mathrm{~nm})$ and high-energy $(650 \mathrm{~nm})$ contributions [see Figure 5(a) and Figure 5(b), respectively] already gives relevant information about the emission centers that yield the observed luminescence. The stretched exponential function has typically been ascribed to (i) a dispersion in excitonic recombination times originated by a broad NC size distribution, each NC size population exhibiting different characteristic decay times, ${ }^{[30,31]}$ (ii) the interaction between neighboring NCs that induce effects related to electron-phonon coupling, ${ }^{[32,33]}$ or (iii) the degree of dispersion from the electron and hole motion due to disorder within the material. ${ }^{[34,35]}$ In these three different but correlated frames, the stretching parameter $\beta$ can be interpreted as a dispersion from the ideal case $(\beta=1)$ of size mono-population of noninteracting (i.e., separated enough) Si NCs. These characteristics allow explaining the observed PL results so far. Indeed, and as can be seen in Figure 5(c) and Figure 5(d), the highenergy contribution presents remarkably lower $\beta$ values than the low-energy one. This occurrence could be attributed to highly localized defect levels at the $\mathrm{Si}_{-} \mathrm{SiO}_{2}$ interface, with a larger degree of disorder than in the case of the high-energy contribution, in agreement with 


\section{WILEY-VCH}

the core-shell model. This assumption is supported by the shorter recombination times $\left(\tau_{2}\right)$, by about one order of magnitude, which might indicate exciton instability because of insufficient carrier confinement due to high disorder. Finally, Figure 5(c) and Figure 5(d) show how $\tau_{2}$ clearly increases for the low-energy contribution at thicker SRO layers until saturating at the thickest $L_{\mathrm{SRO}}=4.7 \mathrm{~nm}$, since even thicker barriers will not propitiate the precipitation and crystallization of larger NCs, but instead the broadening of the NC size distribution centered around a saturated NC mean size. ${ }^{[36]}$ In the case of the high-energy component of PL, we observed the opposite trend compared to the low-energy one, with a reduction of the radiative time $\tau_{2}$ at larger NCs, in agreement with previous reports in similar samples, which indicates luminescence from the amorphous phase at large NCs.

\section{Conclusions}

To summarize, the results obtained from the different structural and optical techniques employed in this study revealed the presence of two different optically active luminescent centers within our EBE-fabricated samples: size-dependent excitonic recombination typically reported for Si quantum dots (low-energy PL contribution) and highly-localized disorderbased emission (high-energy PL component) tightly related to the NC amorphous shell and/or $\mathrm{NC} / \mathrm{SiO}_{2}$ matrix interface defects. Therefore, by means of the EBE deposition of alternated $\mathrm{SiO}_{2}$ and oxidized $\mathrm{Si}$ (i.e., the $\mathrm{SRO}$ ) nanolayers and after a proper high-temperature annealing treatment, we have shown that we are able to tune the luminescence yielded by Si NCs. This has been achieved by controlling the deposited SRO thickness and annealing conditions, which results in the modulation of the two PL emission components. Consequently, the adequacy of the EBE technique to prepare high-quality and light-emitting Si nanostructures has been proved, which paves the way for easy-manufacturing Si NC-based optoelectronic devices.

\section{Experimental section}




\section{WILEY-VCH}

Multilayers of $\mathrm{SRO} / \mathrm{SiO}_{2}$ were deposited onto either $p$-type (100)-Si or fused silica substrates by means of electron-beam evaporation. The employed system was a PFEIFFER VACUUM Classic 500 with a Ferrotec GENIUS electron-beam controller equipped with a Ferrotec CARRERA high-voltage power supply. Before the deposition, the substrates were successively cleaned with acetone, isopropyl alcohol, ethanol and deionized water, being agitated ultrasonically in each process. The MLs were grown depositing alternatively $\mathrm{SiO}_{2}$ and SRO layers, by respectively evaporating $\mathrm{SiO}_{2}$ and $\mathrm{Si}$ targets, both at a rate of $0.5 \AA / \mathrm{s}$. The base pressure in the chamber was $2 \times 10^{-6}$ mbar and the substrate was kept at $100{ }^{\circ} \mathrm{C}$. An $\mathrm{O}_{2}$ line $(1.5 \mathrm{sccm})$, equivalent to $6 \times 10^{-5} \mathrm{mbar}$, was introduced in the chamber during the whole process, avoiding a possible substoichiometry of the $\mathrm{SiO}_{2}$ and promoting a partial oxidation of the $\mathrm{Si}(\mathrm{SRO})$. In order to control the deposition of $\mathrm{SRO}$ and $\mathrm{SiO}_{2}$ layers, nominal and final thicknesses were compared by fabricating a test sample, which consists of two multilayer stacks separated by 10 -nm-thick $\mathrm{SiO}_{2}$ as a reference layer. The first stack (close to the substrate) presents a variation of the SRO layers keeping constant the $\mathrm{SiO}_{2}$ barriers thickness at $2.0 \mathrm{~nm}$, whereas for the other one the $\mathrm{SiO}_{2}$ barrier thickness was varied while keeping constant the SRO layers thickness at $2.0 \mathrm{~nm}$. For the study of the optical properties of EBEprepared Si NCs, different samples were deposited with SRO nominal layers ranging from 1.0 $\mathrm{nm}$ to $2.5 \mathrm{~nm}$ with a constant $\mathrm{SiO}_{2}$ barrier with a nominal thickness of $1.5 \mathrm{~nm}$. This multilayered structure was repeated 5 times, adding a $10-\mathrm{nm}$ of $\mathrm{SiO}_{2}$ as buffer and capping layers. All ML samples underwent an annealing process inside a tubular furnace for $1 \mathrm{~h}$ in $\mathrm{N}_{2}$ atmosphere, at different annealing temperatures ranging from $1000{ }^{\circ} \mathrm{C}$ to $1200{ }^{\circ} \mathrm{C}$.

In order to determine the stoichiometry of the $\mathrm{SRO}$ and $\mathrm{SiO}_{2}$ layers, an XPS analysis was carried out using a PHI 5500 Multitechnique System. Bulk samples of both materials, SRO and $\mathrm{SiO}_{2}$, were required due to the impossibility of the system to distinguish each individual layer in a multilayered structure with nanometer-thick layers. XPS measurements were carried 


\section{WILEY-VCH}

out in 200-nm thick samples (either $\mathrm{SRO}$ or $\mathrm{SiO}_{2}$ ) at a depth of about $50 \mathrm{~nm}$, in order to ensure that information only from the bulk of the samples is acquired, with negligible contribution from their surfaces. The structural characterization was carried out by means of TEM. A conventional mechanical polishing method was employed in cross-section geometry with a subsequent low angle $\mathrm{Ar}^{+}$ion milling up to the electron transparency of the sample. TEM images were acquired by a JEOL $2010 \mathrm{~F}$ microscope, operated at $200 \mathrm{kV}$ and equipped with a field emission gun coupled to a Gatan imaging filter with $0.8 \mathrm{eV}$ energy resolution. EFTEM in scanning mode and HRTEM were employed to analyze the ML structure and the Si NC formation, respectively. The crystallinity of the Si NCs deposited onto fused silica substrate was analyzed by Raman measurements in backscattering configuration with a Horiba Jobin-Yvon LabRam spectrometer, exciting the samples with the 532-nm line of a Nd:YAG laser at room temperature, keeping the power density on the sample at $\sim 5 \times 10^{4}$ $\mathrm{W} / \mathrm{cm}^{2}$. The optical properties were determined measuring the photoluminescence emission of the films grown onto $\mathrm{Si}$ substrate in a range between $550 \mathrm{~nm}$ and $1050 \mathrm{~nm}$, exciting the samples with the $325-\mathrm{nm}$ line of a He-Cd laser, using a power density of $8 \times 10^{3} \mathrm{~W} / \mathrm{cm}^{2}$, and collecting the emitted light with the same experimental setup; the PL intensity of all spectra was corrected by the spectral response of the system. Finally, time-resolved PL measurements were carried out by exciting the samples with the third harmonic line $(355 \mathrm{~nm})$ of a BRILLIANT $\mathrm{Nd}^{3+}$ :YAG 5-ns-pulsed laser at a peak power density of $10^{8} \mathrm{~W} / \mathrm{cm}^{2}$, while inspecting their emission at $650 \mathrm{~nm}$ and $850 \mathrm{~nm}$, in order to understand the origin of the different luminescence contributions.

\section{Acknowledgements}

This work was financially supported by the Spanish Ministry of Economy and Competitiveness (TEC2016-76849-C2-1-R and MAT2015-71035-R). O.B. also acknowledges the subprogram "Ayudas para Contratos Predoctorales para la Formacióon de Doctores" from the Spanish Ministry of Economy and Competitiveness for economical 


\section{WILEY-VCH}

support. J.L.F. also acknowledges the subprogram "Ayudas para la Formación de Profesorado Universitario" from the Spanish Ministry of Education, Culture and Sports for economical support.

Received: ((will be filled in by the editorial staff))

Revised: ((will be filled in by the editorial staff)) Published online: ((will be filled in by the editorial staff))

\section{References}

[1] M. Zacharias, J. Bläsing, P. Veit, L. Tsybeskov, K. Hirschman, and P.M. Fauchet, Appl. Phys. Lett. 1999, 74, 2614.

[2] J. Heitmann, R. Scholz, M. Schmidt, and M. Zacharias, J. Non. Cryst. Solids 2002, 299$302,1075$.

[3] J. Heitmann, F. Müller, M. Zacharias, and U. Gösele, Adv. Mater. 2005, 17, 795.

[4] N. Lalic and J. Linnros, J. Appl. Phys. 1996, 80, 5971.

[5] S. Chan and P.M. Fauchet, Appl. Phys. Lett. 1999, 75, 274.

[6] J. López-Vidrier, Y. Berencén, S. Hernández, O. Blázquez, S. Gutsch, J. Laube, D. Hiller, P. Löper, M. Schnabel, S. Janz, M. Zacharias, and B. Garrido, J. Appl. Phys. 2013, 114, 163701.

[7] J. López-Vidrier, S. Gutsch, O. Blázquez, D. Hiller, J. Laube, R. Kaur, S. Hernández, B. Garrido, and M. Zacharias, Appl. Phys. Lett. 2017, 110, 203104.

[8] I. Perez-Wurfl, X. Hao, A. Gentle, D.-H. Kim, G. Conibeer, and M.A. Green, Appl. Phys. Lett. 2009, 95, 153506.

[9] D. Di, I. Perez-Wurfl, G. Conibeer, and M.A. Green, Sol. Energy Mater. Sol. Cells 2010, 94, 2238.

[10] G. Conibeer, M. Green, R. Corkish, Y. Cho, E.C. Cho, C.W. Jiang, T. Fangsuwannarak, E. Pink, Y. Huang, T. Puzzer, T. Trupke, B. Richards, A. Shalav, and K. lung Lin, Thin Solid Films 2006, 511-512, 654.

[11] S. Tiwari, F. Rana, H. Hanafi, A. Hartstein, E.F. Crabbé, and K. Chan, Appl. Phys. Lett. 1996, 68, 1377.

[12] T.Z. Lu, M. Alexe, R. Scholz, V. Talalaev, R.J. Zhang, and M. Zacharias, J. Appl. Phys. 2006, 100, 014310.

[13] J. López-Vidrier, S. Hernández, D. Hiller, S. Gutsch, L. López-Conesa, S. Estradé, F. Peiró, M. Zacharias, and B. Garrido, J. Appl. Phys. 2014, 116, 133505.

[14] J. López-Vidrier, Y. Berencén, S. Hernández, B. Mundet, S. Gutsch, J. Laube, D. Hiller, P. Löper, M. Schnabel, S. Janz, M. Zacharias, and B. Garrido, Nanotechnology 2015, 26, 185704.

[15] Y. Lebour, P. Pellegrino, S. Hernández, A. Martínez, E. Jordana, J.-M. Fedeli, and B. Garrido, Phys. E 2009, 41, 990.

[16] K. Ma, J.Y. Feng, and Z.J. Zhang, Nanotechnology 2006, 17, 4650.

[17] M. Perálvarez, J. Barreto, J. Carreras, A. Morales, D. Navarro-Urrios, Y. Lebour, C. Domínguez, B. Garrido, M. Peralvarez, C. Dominguez, and C. Domínguez-Horna, Nanotechnology 2009, 20, 1.

[18] A. Martínez, S. Hernández, P. Pellegrino, O. Jambois, P. Miska, M. Grün, H. Rinnert, M. Vergnat, V. Izquierdo-Roca, J.M. Fedeli, and B. Garrido, Phys. Status Solidi C 2011, 8, 969. [19] D.C. Gunduz, A. Tankut, S. Sedani, M. Karaman, and R. Turan, Phys. Status Solidi C 2015, 12, 1229.

[20] S. Alkis, A.K. Okyay, and B. Ortaç, J. Phys. Chem. C 2012, 116, 3432. 


\section{WILEY-VCH}

[21] A. Mehonic, M. Buckwell, L. Montesi, L. Garnett, S. Hudziak, S. Fearn, R. Chater, D. McPhail, and A.J. Kenyon, J. Appl. Phys. 2015, 117, 124505.

[22] S. Hernández, A. Martínez, P. Pellegrino, Y. Lebour, B. Garrido, E. Jordana, and J.M. Fedeli, J. Appl. Phys. 2008, 104, 044304.

[23] M. Zacharias and P. Streitenberger, Phys. Rev. B 2000, 62, 8391.

[24] S. Hernández, J. López-Vidrier, L. López-Conesa, D. Hiller, S. Gutsch, J. Ibáñez, S.

Estradé, F. Peiró, M. Zacharias, and B. Garrido, J. Appl. Phys. 2014, 115, 203504.

[25] J. Ibáñez, S. Hernández, J. López-Vidrier, D. Hiller, S. Gutsch, M. Zacharias, A. Segura, J. Valenta, and B. Garrido, Phys. Rev. B 2015, 92, 035432.

[26] M. Greben, P. Khoroshyy, X. Liu, X. Pi, and J. Valenta, J. Appl. Phys. 2017, 122, 034304.

[27] J.A. Rodríguez, M.A. Vásquez-Agustín, A. Morales-Sánchez, and M. Aceves-Mijares, J. Nanomater. 2014, 2014, 1.

[28] O. Blázquez, J. López-Vidrier, L. López-Conesa, M. Busquets-Masó, S. Estradé, F. Peiró, S. Hernández, and B. Garrido, J. Appl. Phys. 2016, 120, 135302.

[29] G.R. Lin, C.J. Lin, and K.C. Yu, J. Appl. Phys. 2004, 96, 3025.

[30] J. Linnros, N. Lalic, A. Galeckas, and V. Grivickas, J. Appl. Phys. 1999, 86, 6128.

[31] M. Dovrat, Y. Goshen, J. Jedrzejewski, I. Balberg, and a. Sa'ar, Phys. Rev. B 2004, 69, 1.

[32] S.L. Brown, R. Krishnan, A. Elbaradei, J. Sivaguru, M.P. Sibi, and E.K. Hobbie, AIP

Adv. 2017, 7, 055314.

[33] S.L. Brown, J.B. Miller, R.J. Anthony, U.R. Kortshagen, A. Kryjevski, and E.K. Hobbie, ACS Nano 2017, 11, 1597.

[34] L. Pavesi and M. Ceschini, Phys. Rev. B 1993, 48, 17625.

[35] L. Pavesi, J. Appl. Phys. 1996, 80, 216.

[36] J. López-Vidrier, S. Hernández, A.M. Hartel, D. Hiller, S. Gutsch, P. Löper, L. López-

Conesa, S. Estradé, F. Peiró, M. Zacharias, and B. Garrido, Energy Procedia 2011, 10, 43. 

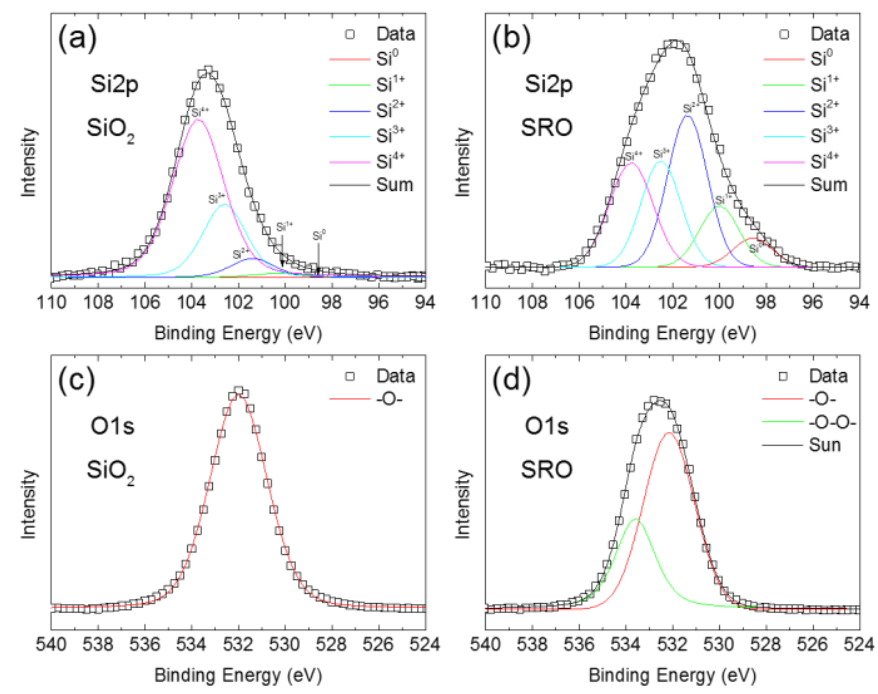

Figure 1. XPS analysis corresponding to bulk deposition of both $\mathrm{SiO}_{2}$ and $\mathrm{SRO}$, after annealing at $1200{ }^{\circ} \mathrm{C}$, employed for the fabrication of the multilayered structure. (a) and (b) present the $\mathrm{Si} 2 \mathrm{p}$ signal of $\mathrm{SiO}_{2}$ and $\mathrm{SRO}$, respectively, deconvolved by means of pseudoVoigt fits related to the different oxidation states of Si. (c) and (d) show the spectra of the O1s for both layers, $\mathrm{SiO}_{2}$ and $\mathrm{SRO}$, respectively. In this case, (c) displays a single pseudo-Voigt fit (-O-), whereas (d) is deconvolved into two pseudo-Voigt contributions related to (-O-) and ($\mathrm{O}-\mathrm{O}-)$ oxygen configurations. 


\section{WILEY-VCH}
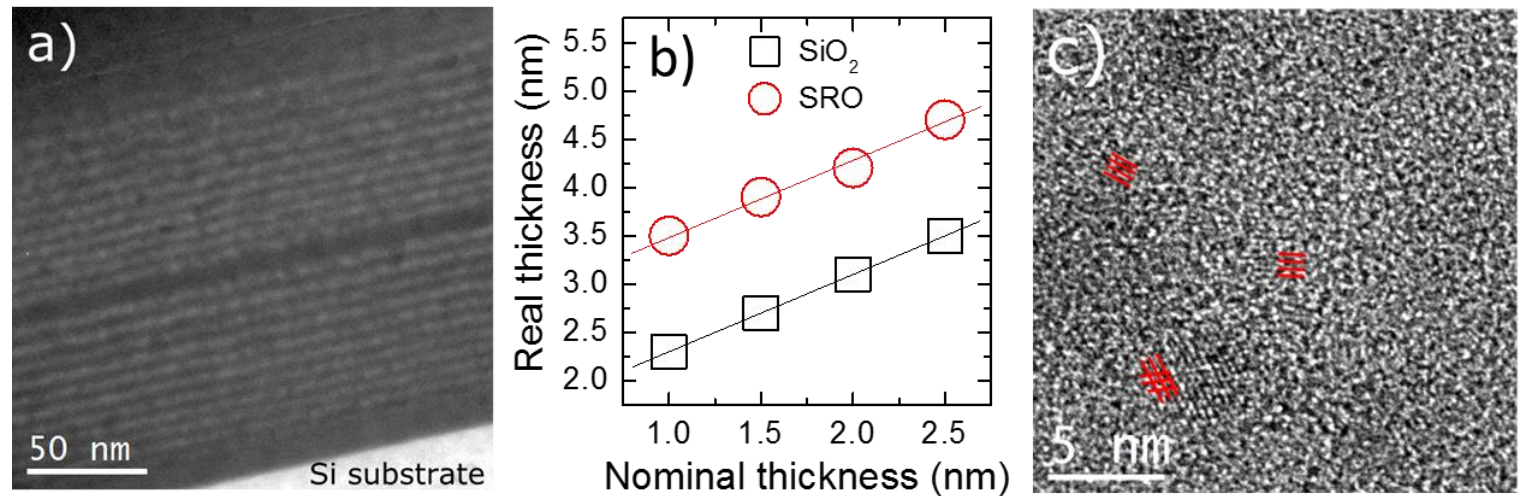

Figure 2. (a) EFTEM image obtained after filtering around the $\mathrm{Si}$ plasmon peak $(\sim 17 \mathrm{eV})$ with a $3 \mathrm{eV}$ energy window. Bright regions in the image correspond to Si-rich oxide (SRO) regions. (b) Relation between nominal and real deposition thicknesses of $\mathrm{SiO}_{2}$ and $\mathrm{SRO}$ layers. (c) HRTEM image in the center of the first stack. Si lattice planes can be observed (highlighted by red lines) for some crystalline nanoaggregates. 

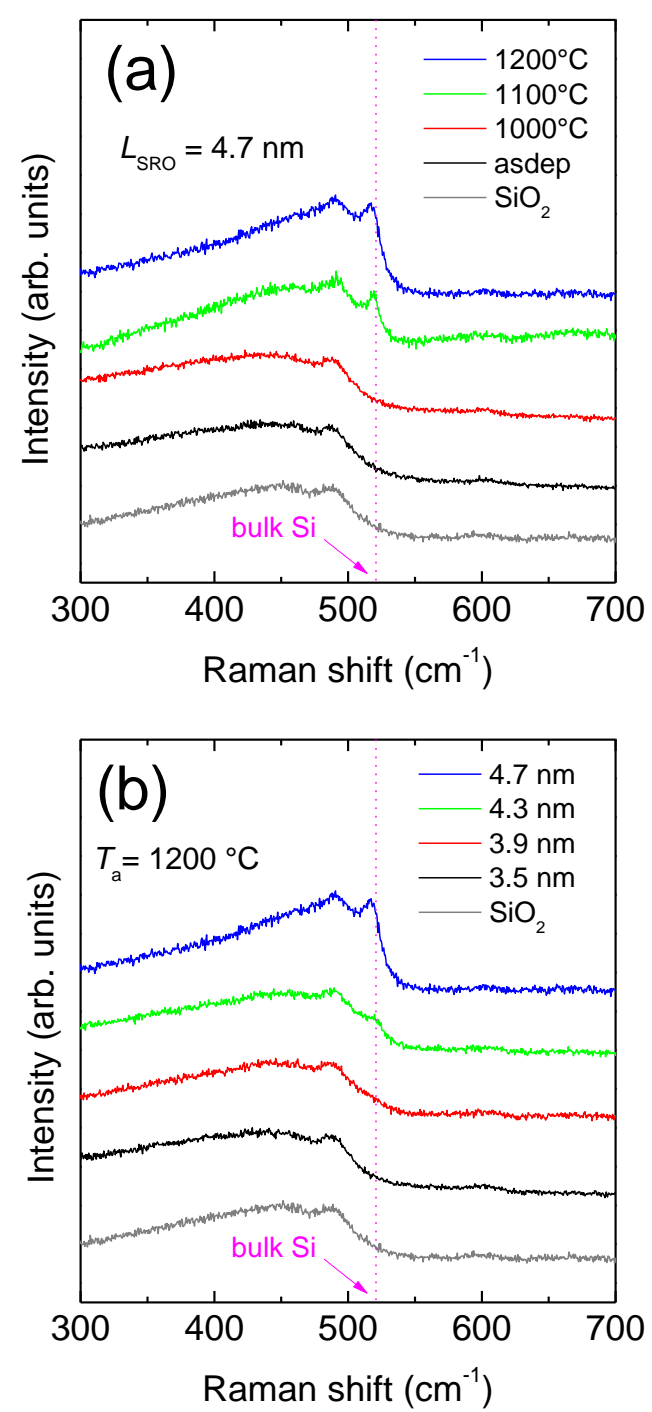

Figure 3. (a) Raman spectra corresponding to the samples with 4.7-nm-thick SRO layers for both the as-deposited samples and the ones annealed at different $T_{\mathrm{a}}$. (b) Raman spectra corresponding to the samples with different SRO thicknesses annealed at $T_{\mathrm{a}}=1200{ }^{\circ} \mathrm{C}$. In both figures, the pink dotted vertical line indicates the peak position of the bulk crystalline $\mathrm{Si}$ signal. In addition, the Raman spectrum of the $\mathrm{SiO}_{2}$ substrate (grey lines) is added for comparison. 


\section{WILEY-VCH}
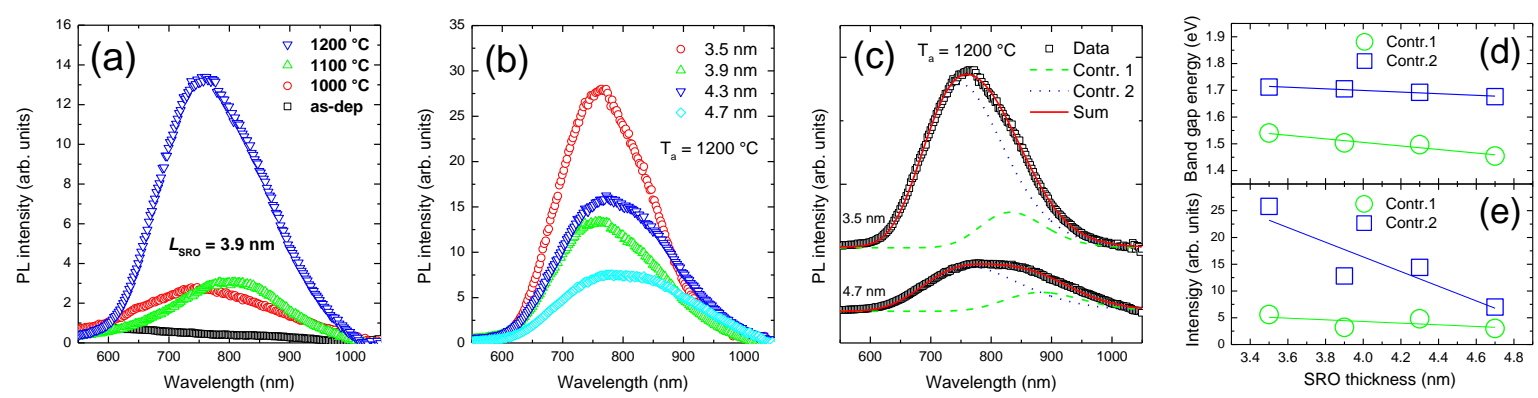

Figure 4. PL spectra corresponding to the samples with (a) constant $L_{\mathrm{SRO}}=3.9 \mathrm{~nm}$ and annealed at different temperatures, and (b) different $L_{\mathrm{SRO}}$ and annealed at $1200{ }^{\circ} \mathrm{C}$. (c) Example of the two-contribution spectra deconvolution carried out on the samples with $L_{\mathrm{SRO}}$ $=3.5$ (thinnest sample) and $L_{\mathrm{SRO}}=4.7 \mathrm{~nm}$ (thickest one), both annealed at $1200{ }^{\circ} \mathrm{C}$. (d) Peak energy corresponding to each PL contribution from the samples annealed at $1200{ }^{\circ} \mathrm{C}$, as a function of $L_{\mathrm{SRO}}$ 
WILEY-VCH
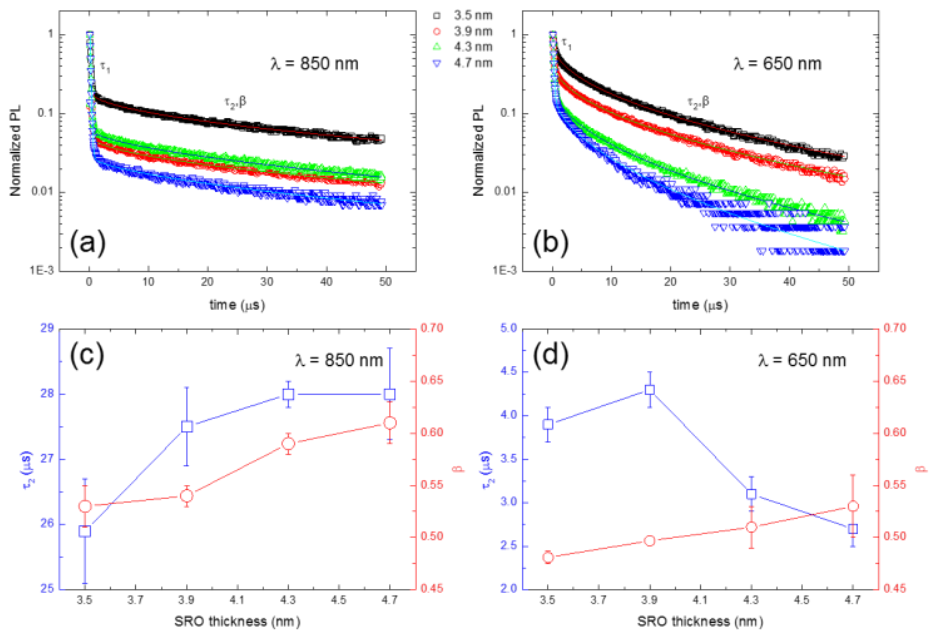

Figure 5. Time evolution of the PL intensity corresponding to the samples containing different $L_{\text {SRO }}$ and annealed at $1200{ }^{\circ} \mathrm{C}$, measured at (a) $850 \mathrm{~nm}$ and (b) $650 \mathrm{~nm}$. The corresponding fits to the fast and slow components are also displayed. (c) and (d) plot the slow decay time, $\tau_{2}$, and stretching parameter, $\beta$, corresponding to the same samples in (a) and (b). 


\section{WILEY-VCH}

Table 1. Peak positions corresponding to the different contributions of Si2p and O1s, obtained fitting the XPS spectra acquired from $\mathrm{SiO}_{2}$ and $\mathrm{SRO}$ layers.

\begin{tabular}{lcccc}
\hline & \multicolumn{2}{c}{$\mathrm{SiO}_{2}$} & \multicolumn{2}{c}{ SRO } \\
\cline { 2 - 5 } & $\mathrm{Si} 2 \mathrm{p}$ & $\mathrm{O} 1 \mathrm{~s}$ & $\mathrm{Si} 2 \mathrm{p}$ & $\mathrm{O} 1 \mathrm{~s}$ \\
\hline Peak position (eV) & - & & 98.6 & \\
& 100.1 & 532.0 & 100.0 & 532.1 \\
& 101.4 & - & 101.3 & 533.6 \\
& 102.6 & & 102.5 & \\
Integrated area & 103.7 & 34.90 & 103.7 & 17.54 \\
(arb. units) & 7.25 & & 4.92 & \\
Atomic concentration & 34 & 66 & 42 & 58 \\
$(\%)$ & & & & \\
\hline
\end{tabular}

